\title{
ECO-friendly Distributed Routing Protocol for Reducing Network Energy Consumption
}

\author{
Daisuke Arai and Kiyohito Yoshihara \\ KDDI R\&D Laboratories Inc. \\ 2-1-15 Ohara Fujimino-shi Saitama, Japan \\ Email: \{di-arai, yosshy\}@kddilabs.jp
}

\begin{abstract}
The growth of Internet traffic has led to an increase in energy consumption by network equipment such as routers and switches. Consequently, energy consumption is becoming a key environmental, social, political and cost issue. Focusing on energy consumption by Internet Service Provider networks, the energy bottleneck is routers. Thus, it is imperative for us to reduce the energy consumption of routers. In this paper, we propose a new ECO-friendly distributed routing protocol (ECO-RP) based on OSPF. In our proposal, an ECO-RP entity periodically checks the amount of traffic forwarded by the ECORP entity. Moreover, an ECO-RP entity floods information on the amount of traffic to other entities such as the OSPF Link State Advertisement. This allows each ECO-RP entity to obtain information on traffic in the network, and to dynamically change OSPF link weights based on the information. For example, when the amount of overall network traffic is small, the entity changes link weights so that the traffic can be routed only through a subset of routers, and unneeded routers can shift into sleep mode. Several simulation studies, which assume two ECO-RP enabled networks, are conducted. The result shows that the proposed protocol is able to reduce energy consumption by about $18.5 \%$ at maximum without network congestion.
\end{abstract}

\section{INTRODUCTION}

Internet traffic is increasing rapidly, and it has almost doubled each year since 1997 [1]. Energy consumption by network equipment such as routers and switches to carry this traffic is also increasing [2], [3]. Consequently, energy consumption is becoming a key environmental, social, political and cost issue. When we focus on energy consumption by Internet Service Provider (ISP) networks, the energy bottleneck is routers [4], [5]. Thus, it has become imperative to reduce the energy consumption of routers.

Research and development aiming at reducing network energy consumption [6], [7], [8], [9], [10], [11] has been carried out. Some research has focused on the extension of network equipments which change a link speed based on the utilization of the link [6]. Still other research has examined the extension of network equipments which can be put into sleep mode when no packets are buffered [7], [8], [9]. Other researches have shown how to reconfigure a network in order to reduce the number of routers in use [10], [11]. To reconfigure a network, a centralized network management scheme is shown in [11]. However, when we focus on the energy consumption of routers in an ISP network, it is difficult to reduce energy consumption solely by applying these systemoriented schemes [6], [7], [8], [9], because routers in an ISP network must forward user traffic constantly. Furthermore, to reduce the energy consumption of routers by means of these centralized network management schemes [10], [11], a network operator must install servers. In some cases, installing servers may be difficult from a cost perspective.

Changing the network configuration based on the amount of traffic has the potential to reduce the energy consumption of routers, and achieving this using a distributed scheme is promising in terms of cost. In this paper, as a solution to this, we propose a new ECO-friendly distributed routing protocol (ECO-RP) based on Open Shortest Path First (OSPF) [12]. We introduce ECO-RP entities to a network. An ECO-RP entity periodically checks the amount of traffic forwarded by the entity. In addition, an ECO-RP entity floods information on the amount of traffic to other entities such as the OSPF Link State Advertisement (LSA). Thereby, each ECO-RP entity is able to obtain information on the traffic in a network. Each ECORP entity may change OSPF link weights dynamically based on the information. For example, when the amount of overall network traffic is small, the entity changes link weights so that the traffic can be routed only through a subset of routers, and unneeded routers can shift into sleep mode. To evaluate the performance of the proposed protocol from the viewpoint of link utilization, energy consumption and maximum hop count, we simulate network behavior on two networks which are generated by a well-known topology generator.

This paper is organized as follows: in Sect. II, we present a network model and its corresponding energy consumption model, which we focus on throughout the paper. We review related work in Sect. III. In Sect. IV, we propose an ECOfriendly distributed routing protocol. We simulate the network behavior of networks when we deploy the proposed protocol in Sect. V. Finally, in Sect. VI, we evaluate the proposed protocol in terms of link utilization, energy consumption and maximum hop count.

\section{Network AND ENERGy CONSUMPtion MOdEL}

We present a network model and its corresponding energy consumption model, which we focus on throughout the paper.

\section{A. Network model}

We consider an ISP network where an OSPF routing protocol is running. It is composed of a number of nodes (routers). Let $N$ denote the set of nodes $n$, and $L$ denote the set of links 
$l$. We use the notion $(i, j)$ in place of link $l$ from node $i$ to node $j$. The capacity of link $l$ is denoted by $b_{l}$. The set of ingress-egress (IE) pairs $k=\left(s_{k}, t_{k}\right)$ is denoted by $K$ with $s_{k}$ referring to the ingress node and $t_{k}$ referring to the egress node of IE pair $k$. Let $P_{k}$ denote the set of all possible paths $p$ from node $s_{k}$ to $t_{k}$. We use notion $l \in p$ if link $l$ belongs to a path $p$. The traffic demand of IE pair $k$ is denoted by $d_{k}$. In addition, the link load of link $l$ is denoted by $l l_{l}$.

Each link $l$ is associated with a weight $w_{l}$ and the traffic is carried along shortest paths. Let $P_{k}^{S P}$ denote the set of shortest paths (SP) from node $s_{k}$ to $t_{k}$ with respect to the link weight $w_{l}$, thus $P_{k}^{S P}$ is defined as:

$$
P_{k}^{S P}=\left\{p \in P_{k} \mid \sum_{l \in p} w_{l}=\min _{p^{\prime} \in P_{k}} \sum_{l^{\prime} \in p^{\prime}} w_{l^{\prime}}\right\}
$$

In each node $i$, the incoming traffic with the same destination $t$ is aggregated and then split to links $(i, j)$ that belong to one of the shortest paths of the IE pair $(i, t)$. Let $\phi_{i j}^{t}$ denote the corresponding splitting ratio. Thus, $\phi_{i j}^{t}$ refers to the fraction of overall traffic passing through node $i$ and destined to node $t$ that is forwarded on link $(i, j)$. It is usually assumed that these splitting ratios $\phi_{i j}^{t}$ are equal, thus $\phi_{i j}^{t}$ is modeled [13] as :

$$
\begin{gathered}
\phi_{i j}^{t}=\frac{1}{\mid\left\{j^{\prime}:\left(i, j^{\prime}\right) \in p \text { for some } p \in P_{(i, t)}^{S P}\right\} \mid} \\
\sum_{j:(i, j) \in p \text { for some }{ }_{p \in P_{(i, t)}^{S P}}} \phi_{i j}^{t}=1
\end{gathered}
$$

Figure 1 shows a typical ISP network which is composed of a number of nodes. These nodes can be categorized as either a backbone node or an access node. Backbone nodes provide an intermediate layer over access nodes. Meanwhile, access nodes connect ISP customers or neighboring networks to provide the Internet service. The traffic is generated at the ISP customer or the neighboring network and it comes into the ISP network. Moreover, this traffic is routed to the other ISP customer or the other neighboring network through access and backbone nodes. In other words, only the access node can be an ingress and/or egress node. The traffic demand $d_{k}$ changes periodically [14], [15]. Let $d_{k}(t)$ and $l l_{l}(t)$ denote the traffic demand and the link load at time $t$, respectively.

As an example, we show the details of node behavior with the traffic demand $d_{(1,4)}$ when all links are associated with the same weight. To forward traffic, ingress node 1 calculates shortest paths from itself to egress node 4 by using Eq. 1. Since node 1 has two shortest paths (path 1-2-4 and path 1-3-4), node 1 splits traffic demand $d_{(1,4)}$ to link $(1,2)$ and link $(1,3)$ according to the splitting ratio $\phi_{12}^{4}$ and $\phi_{13}^{4}$. Splitting ratio $\phi_{12}^{4}$ and $\phi_{13}^{4}$ are calculated by using Eq. 2 and Eq. 3. The link load $l l_{(1,2)}$ by the traffic demand $d_{(1,4)}$ is $\phi_{12}^{4} d_{(1,4)}=0.5 d_{(1,4)}=$ $l l_{(1,2)}$, and the link load $l l_{(1,3)}$ by the traffic demand $d_{(1,4)}$ is $\phi_{13}^{4} d_{(1,4)}=0.5 d_{(1,4)}=l l_{(1,3)}$. In the other node, to forward traffic, the node calculates shortest paths from itself to the egress node, and forwards received traffic in the same way as node 1.

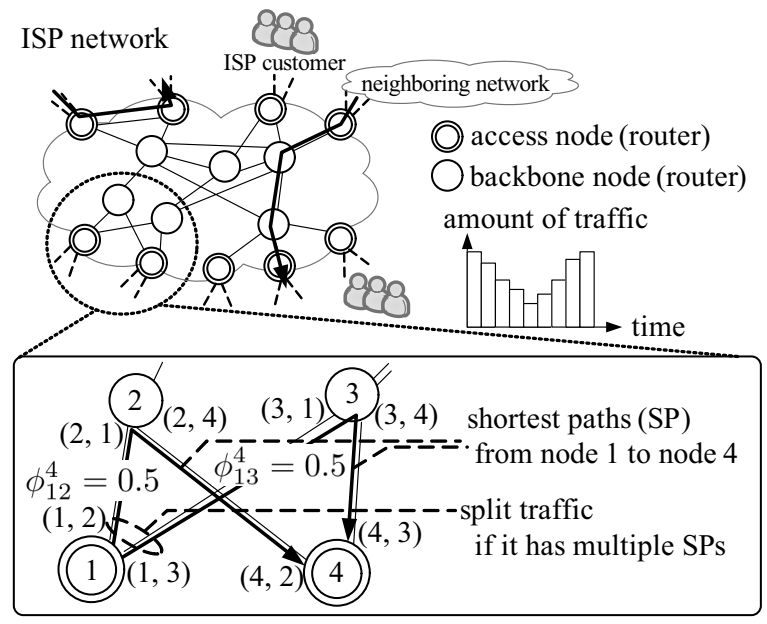

Fig. 1. Network model

\section{B. Energy consumption model}

A node is composed of one or more chassis, in which one or more line cards are installed. Energy consumption of a node is determined by the sum of energy consumption of chassis and line cards. Moreover, energy consumption of chassis and line cards is determined by product type [10].

Let $c c_{i}$ denote energy consumption of the chassis of node $i$, and let $l c c_{(i, j)}$ denote energy consumption of the line card of link $(i, j)$. The network is composed of a number of nodes, thus network energy consumption $E C$ is modeled as:

$$
E C=\sum_{i}\left\{c c_{i}+\sum_{j} l c c_{(i, j)}\right\}
$$

Therefore, the value of $E C$ is determined by the sum of energy consumption of all chassis and line cards on a network. Our aim is to reduce the value of $E C$.

\section{RELATED WORK}

Research and development aiming at reducing network energy consumption $E C$, has been carried out.

Standard initiatives include IEEE 802.3az Energy Efficient Ethernet (EEE) [6]. It changes the link speed based on the utilization of the link by the extension of network equipment. Other research has introduced an energy-saving mode into LAN switches. The LAN switches can be put to sleep or into an energy saving mode when no packets are buffered [2], [7], [8], [9]. However, reducing the energy consumption of nodes solely by these means is difficult, because every node in ISP networks must forward user traffic constantly, thus it is unlikely that nodes can be put to sleep.

Some research shows that energy consumption could be reduced by changing the network configuration based on the amount of traffic in order to reduce the number of nodes in use [10]. However, specific mechanisms for changing the network configuration have not been given. 
A network reconfiguration scheme [11] has been developed. The scheme introduces a central control server to manage the network configuration, and the server collects traffic information from all nodes. The central control server determines the network configuration in order to reduce the number of nodes in use. However, a network operator must install the central control server. In some cases, installing servers may be difficult from a cost perspective.

For this reason, a distributed scheme with no central server is needed.

\section{Proposal on ECO-Friendly Distributed ROUTING PROTOCOL}

To reduce the $E C$ value of Eq. 4, we focus on periodic change in traffic demand [14], [15] and propose a new ECOfriendly distributed protocol (ECO-RP).

\section{A. Protocol design}

ECO-RP changes the network topology based on the amount of overall network traffic in order to reduce the number of active chassis and line cards, i.e. when the amount of overall network traffic is small, the network topology changes so that traffic can be routed by a subset of nodes and their links, and unneeded chassis and line cards can shift into sleep mode.

To change the network topology in response to the amount of overall network traffic, we propose ECO-RP based on OSPF.

OSPF is the most commonly used intra-domain routing protocol. In OSPF, traffic is routed along the shortest paths as described in Sect. II. The shortest paths are calculated from link weight $w_{l}$ which is assigned by a network operator. Fixed link weights are generally used. By using fixed link weights, the network keeps the same topology except when link failures occur. OSPF can dynamically re-route around link failures; however, changing the paths in response to the amount of traffic is out of the scope.

Unlike OSPF, ECO-RP changes the network topology based on the amount of overall network traffic. We show the behavior of an ECO-RP enabled network in Fig. 2. ECO-RP entities periodically check the amount of traffic forwarded by the ECORP entity. Moreover, an ECO-RP entity floods information on the amount of traffic to other entities. This allows each ECORP entity to obtain the same traffic information in a network. From the traffic information, each ECO-RP entity calculates traffic trends. The traffic trend indicates whether the amount of overall network traffic is trending up or down. An ECO-RP entity changes link weights based on the traffic trend. When the traffic trend is trending downward, the ECO-RP entity changes its link weights so that the traffic can be routed by a subset of routers. Moreover, the ECO-RP entity shifts into sleep mode if it is not required for traffic forwarding (from the left to right of Fig. 2). On the other hand, when traffic is trending upwards, the ECO-RP entity changes link weights so that the traffic can be routed by all routers (from the right to left of Fig. 2).

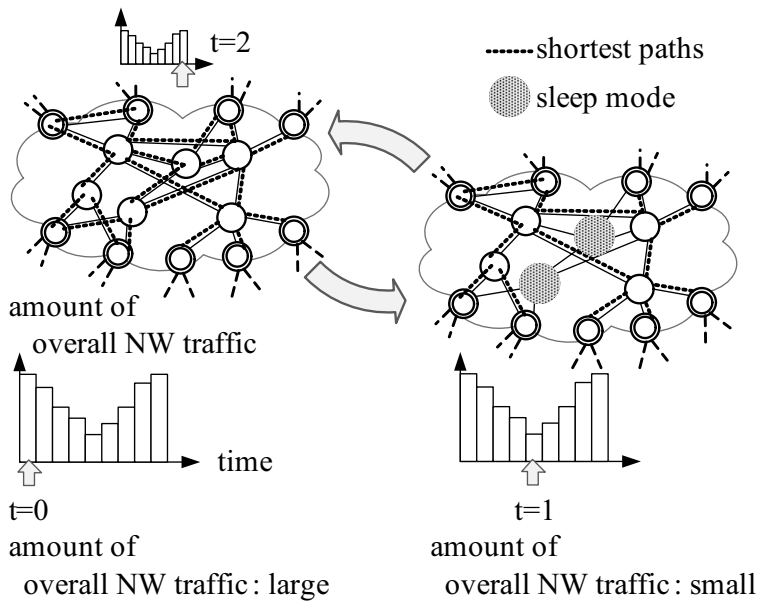

Fig. 2. Behavior of an ECO-RP enabled network

By the aggregation of the traffic, link utilization $l l_{l} / b_{l}$ is changed. If the largest link utilization in the network (maximum link utilization) exceeds 1.0, network congestion occurs, and the performance of the network is significantly decreased. Therefore, the ECO-RP entity should change link weights without network congestion. Moreover, in the standard operation of OSPF, the OSPF entity considers the sleep node as node failure, and removes the information of the sleep node. To resume the sleep node, the ECO-RP entity should distinguish between the sleep node and node failure.

\section{B. Protocol overview}

We propose the following new message and databases as additional OSPF capabilities.

1) Network State Advertisement (NSA). The NSA is a message. An ECO-RP entity periodically checks the amount of traffic forwarded by the ECO-RP entity. Then, the ECO-RP entity floods information on the amount of traffic to other entities by the NSA.

2) Network State Database (NSDB). The NSDB is a database which is formed by collecting NSAs from all nodes. The ECO-RP entity calculates the traffic trend from the NSDB.

3) Historical Link State Database (HLSDB). The HLSDB is a database which is formed by collecting OSPF Link State Advertisements (LSAs). It is the same as an OSPF Link State Database (LSDB) except that the HLSDB contains entries of sleep nodes. The ECO-RP entity distinguishes between sleep nodes and node failure by the HLSDB.

Each ECO-RP entity obtains the same traffic information in a network by the NSA and the NSDB. From the NSDB, each ECO-RP entity calculates traffic trends and changes the network topology. The active ECO-RP entity distinguishes between sleep nodes and node failure by the HLSDB. Moreover, the active ECO-RP entity resumes a sleep node if the sleep node needs to carry the increasing traffic. 


\section{Provisioning}

We make the following provisioning to operate ECO-RP entities.

1) For each ECO-RP entity, a network operator provides a node class that presents an access or backbone node.

2) For each link of the nodes, a network operator assigns a link weight $w_{l}$.

3) Clocks of all ECO-RP entities in a network are synchronized (e.g. by Network Time Protocol (NTP)).

4) A network operator provides the following parameter values. All ECO-RP entities in a network use the same values.

(Param. a) Period to check the amount of traffic

(Param. b) Period to calculate the traffic trend

(Param. c) Scaling factor $\alpha$ to change link weights

(Param. d) Threshold $\beta$ to avoid network congestion

In our proposal, a link weight initially assigned by a network operator is set to a default weight $w_{l_{-} \text {default }}$. For example, a network operator assigns the default weight which is inversely proportional to the link capacity [16]. When the traffic is trending upward, an ECO-RP entity tries to avoid congestion on the network by using the default weight. In contrast, when the traffic trend is trading downward, an ECO-RP entity aggregates traffic to a subset of routers by changing the link weights to reduce the number of active chassis and line cards.

\section{Details on ECO-RP}

We show the behavior of an ECO-RP entity in Fig. 3. The ECO-RP entity performs the following 5 processes:

1) Standard operation of OSPF: Each ECO-RP entity has an OSPF LSA, and it includes the state of the links, such as adjacencies and link weights. The LSA is flooded throughout the network (Fig. 3 (S1)). The collection of LSAs from all nodes forms an OSPF LSDB (Fig. 3 (S2)). An IP routing table is calculated from the LSDB by Eq. 1 (Fig. 3 (S3)).

2) NSA flooding and collection: Each ECO-RP entity monitors the amount of traffic in each period specified by (Param. a) and floods information on the amount of traffic throughout the network by the NSA (Fig. 3 (S4)). All ECO-RP entities flood the NSA at the same time. The flooded NSAs are collected by all ECO-RP entities. Then, an ECO-RP entity stores them in the NSDB (Fig. 3 (S5)). The NSDB includes the NSAs of all nodes and the history of the NSA changes.

3) Traffic trend calculation: All ECO-RP entities can ascertain the historical changes in the amount of overall network traffic from the NSDB, and they can then calculate the traffic trend (Fig. 3 (S6)). After collecting NSAs, all ECO-RP entities calculate the trend by the slope of the linear regression (SLR) of the period specified by (Param.b). The traffic will trend either upwards or downwards. The traffic trend is calculated by the following equation:

$$
\text { traffic trend }=\left\{\begin{array}{l}
\text { uptrend, for } 0 \leq \text { SLR } \\
\text { downtrend, for SLR }<0
\end{array}\right.
$$

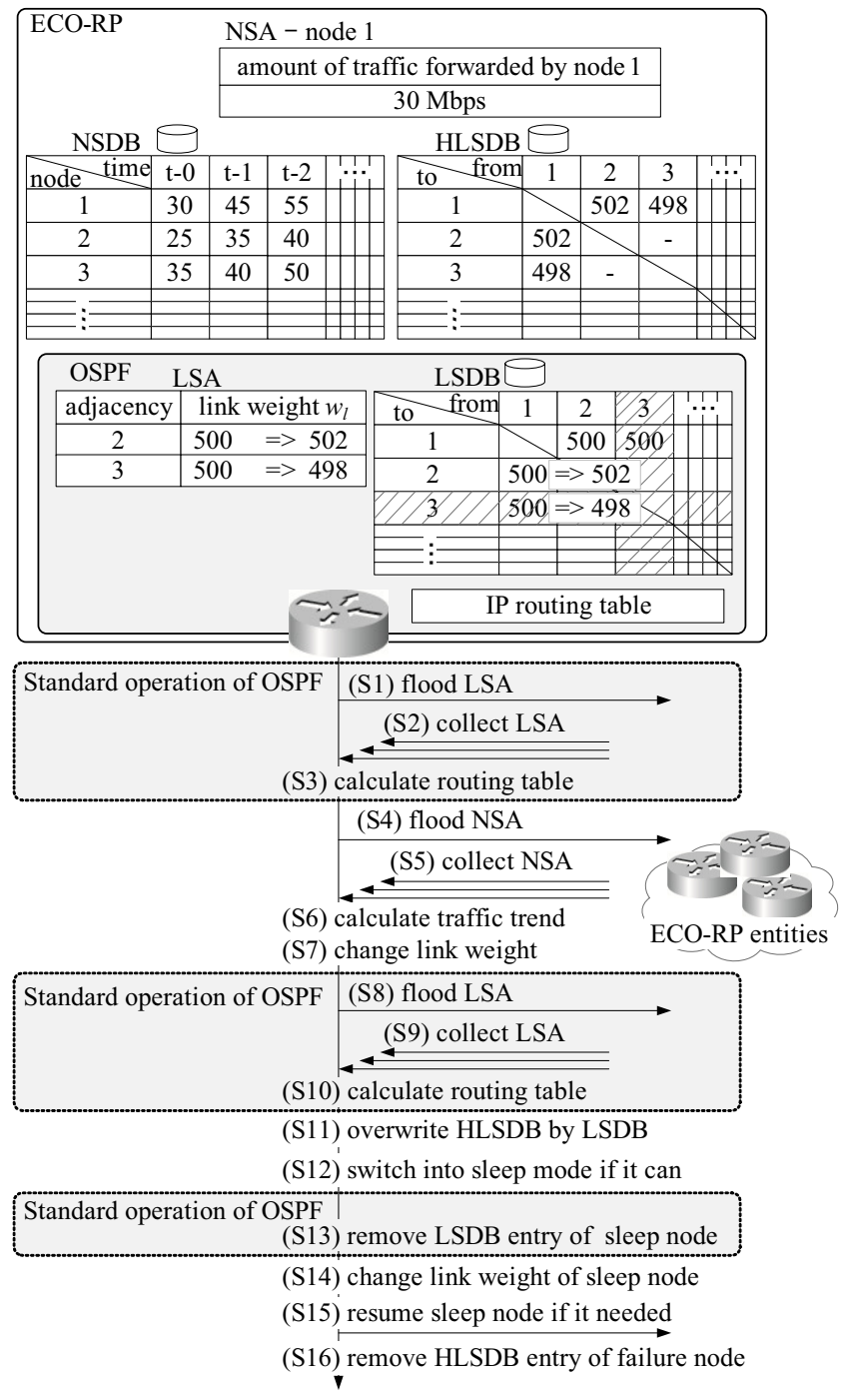

Fig. 3. Behavior of an ECO-RP entity

4) Link weight change: All ECO-RP entities change the link weight $w_{l}$ of the OSPF LSA based on the trend, the SLR, and the $\alpha$ (Param. c) to change the network topology. If the traffic is trending downward, the link weight $w_{l}$ is changed to the new weight $w_{(i, j)}^{\prime}$ as below (Fig. 3 (S7)):

$$
w_{(i, j)}^{\prime}=\left\{\begin{array}{c}
w_{(i, j)}+\mathrm{SLR} \times \alpha, \\
\quad \text { for } i=\text { access node and } j \bmod 2=0 \\
w_{(i, j)}-\mathrm{SLR} \times \alpha, \\
\quad \text { for } i=\text { access node and } j \bmod 2=1 \\
w_{(i, j)}+\mathrm{SLR} \times \alpha, \\
\quad \text { for } i=\text { backbone node and } i \bmod 2=0 \\
w_{(i, j)}-\mathrm{SLR} \times \alpha, \\
\quad \text { for } i=\text { backbone node and } i \bmod 2=1 \\
\quad \text { s.t. } \\
1 \leq w_{(i, j)}^{\prime} \leq 2 w_{(i, j) \_d e f a u l t}-1
\end{array}\right.
$$


If the traffic is trending upward, the link weight $w_{l}$ is changed to the new weight $w_{(i, j)}^{\prime}$ as below:

$$
\begin{gathered}
w_{(i, j)}^{\prime}=\left\{\begin{array}{c}
w_{(i, j)}-\operatorname{SLR} \times \alpha, \\
\text { for } i=\operatorname{access} \text { node and } j \bmod 2=0 \\
w_{(i, j)}-\operatorname{SLR} \times \alpha, \\
\text { for } i=\text { backbone node and } i \bmod 2=0 \\
\text { s.t. }
\end{array}\right. \\
w_{(i, j) \_ \text {default }} \leq w_{(i, j)}^{\prime}
\end{gathered}
$$

If the amount of overall network traffic becomes large when the ECO-RP entity aggregates traffic to a subset of nodes, network congestion might occur. To counter this, if the amount of overall network traffic is larger than $\beta$ (Param. d), the link weight $w_{l}$ is changed to the default link weight $w_{(i, j)} \_$default regardless of the traffic trend.

The new weight $w_{(i, j)}^{\prime}$ changes network topology by the standard operation of OSPF (Fig. 3 (S8), (S9), (S10)).

5) Shift into sleep mode and resume from sleep mode: Each ECO-RP entity overwrites the HLSDB with the LSDB, when the OSPF LSDB is changed (Fig. 3 (S11)). A backbone node shifts into sleep mode when the paths from an access node to another access node are lost (every IE-pair) (Fig. 3 (S12)). In this mode, the backbone node can deactivate all functions and it can shut down. In the standard operation of OSPF, entry of sleep node is removed from the LSDB (Fig. 3 (S13)). Only the HLSDB has the entries of sleep nodes. By comparing the HLSDB and the LSDB, each active ECO-RP entity can ascertain the sleep node, and change the link weight of the sleep node on the HLSDB (Fig. 3 (S14)) by Eq. 6, Eq. 7 and Eq. 8. By calculating shortest paths from the HLSDB, the active ECO-RP entity resumes the sleep node through the network (e.g. by Wake-on-LAN) if the sleep node is on the paths of every IE-pair (Fig. 3 (S15)). In addition, if resumption of the sleep node fails, the active ECO-RP entity identifies its node as a failure node and entry of failure node is removed from the HLSDB (Fig. 3 (S16)).

\section{Simulations}

To evaluate the performance of the proposed protocol from the viewpoint of link utilization, energy consumption, and maximum hop count, we simulate network behavior.

\section{A. Network topologies}

We generate two network topologies, NW1 and NW2 for short, for simulations using the BRITE network topology generator [17]. The parameter settings are shown in Table I. In the generation, we use the Waxman (random network) model
TABLE I

PARAMETERS OF BRITE FOR SIMULATION

\begin{tabular}{c|c|c}
\hline topology & NW1 & NW2 \\
\hline \hline type & \multicolumn{2}{|c}{ Router only } \\
\hline$|N|$ & \multicolumn{2}{|c}{30} \\
\hline router model & Waxman & BA \\
\hline BRITE's $\alpha$ & 0.15 & - \\
\hline BRITE's $\beta$ & 0.2 & - \\
\hline links/node & \multicolumn{2}{|c}{2} \\
\hline$n$ Placement & \multicolumn{2}{|c}{ Random } \\
\hline
\end{tabular}

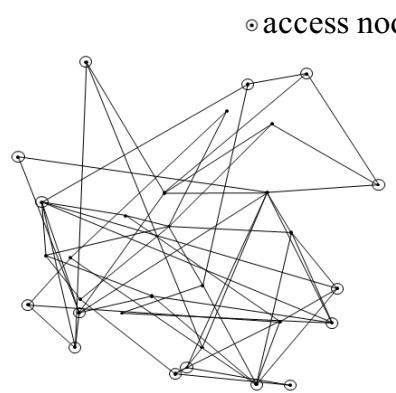

NW1
$|N|=30,|L|=60$

$$
|N|=30,|L|=57
$$$$
|N|=30,|L|=57
$$

NW2

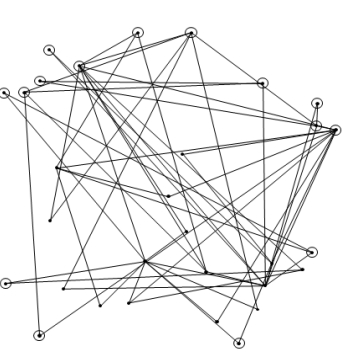

the number of access nodes $=|N| \times 0.5=15$

Fig. 4. Network topologies for simulation

for NW1 and the Barabasi Allbert (BA) model for NW2. Figure 4 shows the two network topologies. To categorize each node into either an access or backbone node, we calculate the Euclidean distance from the center of the network to each node and then we categorize 15 nodes (half of all nodes) as access nodes in descending order of the Euclidean distance.

In the simulations, we consider a same link capacity $b_{l}$, and a same default link weight $w_{l_{\text {_ddefault }}}$ for all links. We set link capacity $b_{l}$ to $1 \mathrm{Gbps}$ and default link weight $w_{l}$ default to 500.

\section{B. Traffic model}

Only the access node can be an ingress and/or egress node as described in Sect. II. Thus the simulated networks can have 15 (the number of access nodes) $\times 14$ (the number of access nodes -1$)=210$ IE-pairs. To consider the traffic demand of each IE-pair $(s k, t k)$, we pick two random numbers $o, r \in[0,1]$ for each node. Let $o_{s k}$ denote a random number $o$ for node $s k$ and $r_{t k}$ denote a random number $r$ for node $t k$. Further, for each IE-pair $(s k, t k)$ we pick a random number $s_{(s k, t k)} \in[0,1]$ [18]. Each traffic demand $d_{(s k, t k)}(t)$ is described as below:

$$
d_{(s k, t k)}(t)=\gamma(t) o_{s k} r_{t k} s_{(s k, t k)} e^{-\delta(s k, t k) / 2 \Delta}
$$

Here, $\delta(s k, t k)$ is the Euclidean distance between $s k$ and $t k$ and $\Delta$ is the largest Euclidean distance between any pair of nodes. Above, the $o_{s k}$ and $r_{t k}$ model the activity level of the node, and a value closer to 1 suggests a node which generates a large amount of traffic. The distance factor $e^{-\delta(s k, t k) / 2 \Delta}$ 


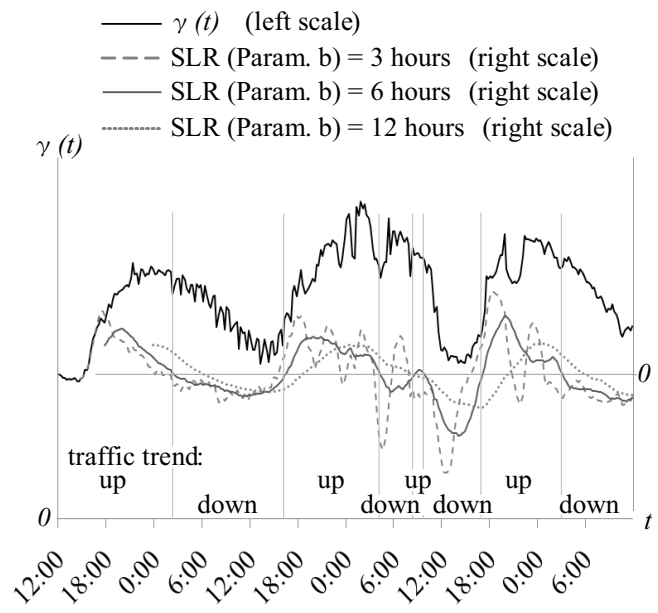

Fig. 5. $\gamma(t)$ for simulation

implies a greater amount of traffic for the closer pair of nodes. Moreover, $\gamma(t)$ is traffic information to describe the amount of traffic at time $t$. In the simulations, we use traffic information of a real network for $\gamma(t)$. Real traffic information of the pan-European research network GEANT has been shown [19]. Traffic information for each 15-minute interval over a 4-month period is available. We use the traffic information from 12:00 noon Thursday 21st of April to 11:45 a.m. Saturday 23rd of April. Figure 5 shows $\gamma(t)$ for the simulations. In addition, we normalize $\gamma(t)$, so that the maximum link utilization $l l_{l} / b_{l}$ is 0.9 when the peak traffic is forwarded by OSPF nodes to fit the real link utilization [19]. If the link utilization $l l_{l} / b_{l}$ is greater than 1.0, it means that network congestion occurs as a result of network topology changes.

\section{Parameter values for ECO-RP entities}

To operate ECO-RP entities, a network operator must provide four parameter values as described in Sect. IV. We set 15 minutes for (Param. a) since we use the traffic information obtained during 15-minute intervals. In real operation, a network operator would decide this parameter value to avoid frequent flooding of NSA.

The ECO-RP entity calculates the traffic trend by the SLR for the period specified by (Param. b) as described in Sect. IV. To decide the value of (Param. b), we calculate the SLR of the traffic information with the variable value of (Param. b) and we show the calculation result in Fig. 5. From the result, we set this parameter to 6 hours, because frequent changes in the traffic trend can be avoided in comparison to the SLR for 3 hours, and it can detect the traffic trend more quickly than the SLR for 12 hours. In real operation, a network operator can decide this parameter by conducting a preliminary survey of the traffic patterns before replacing with ECO-RP enabled nodes.

We simulate network behavior with variable values of (Param. c) and (Param. d), when all the nodes are replaced with ECO-RP enabled ones.

\section{EVALUATIONS}

The ECO-RP should change link weights without network congestion as described in Sect. IV. To evaluate this, we measure the maximum link utilization by the simulation, and Fig. 6 and Fig. 7 show the results. Moreover, we measure the number of active backbone nodes, active links, and the maximum hop count of all paths, to evaluate the performance of ECO-RP from the viewpoint of energy consumption and maximum hop count, and Fig. 8 shows the result. In this evaluation, active nodes and links refer to the nodes and links which forward at least 1 bit traffic.

\section{A. Maximum link utilization}

First, we set $\alpha$ to 0.01 or 0.02 or 0.03 (variable) and set $\beta$ to 1.0 (fixed). Fixed $\beta(=1.0)$ means that the amount of overall network traffic is smaller than $\beta$ over the simulation. Figure 6 shows the maximum link utilization over the simulation. Almost all the time, the maximum link utilization of ECO$\mathrm{RP}$ is greater than that of OSPF. Furthermore, the maximum link utilization of ECO-RP exceeds 1.0 when we set $\alpha$ to 0.03 in NW1 (in the final upward trend at bottom-left of Fig. $6)$, in a similar way, we can see network congestion when we set $\alpha$ to 0.02 or 0.03 in NW2 (in the second downward trend at center-right and bottom-right of Fig. 6). Moreover, the increase in the maximum link utilization varies in response to $\alpha$ (the maximum link utilization is increased by a larger $\alpha$ ). A large $\alpha$ leads to a large amount of link weight change as described in Sect. IV. From this, we can see that a large amount of link weight change leads to greater aggregation of traffic. Therefore, $\alpha$ decides how much to aggregate the traffic. To reduce energy consumption, a network operator would decide this parameter value in order to provide high aggregation without network congestion. On the other hand, network congestion occurs as a result of the high aggregation. To counter this, if the amount of overall network traffic is larger than $\beta$, the ECO-RP entity changes link weight $w_{l}$ to the default link weight $w_{l \_d e f a u l t}$ as described in Sect. IV.

To evaluate the effect of $\beta$, we set $\alpha$ to 0.03 (fixed, network congestion occurs in both NW1 and NW2) and set $\beta$ to 0.6 or 0.7 (variable). Figure 7 shows the maximum link utilization over the simulation. From the simulation result, we can see that the maximum link utilization of ECO-RP falls below 1.0 in NW1 and NW2, unlike the maximum link utilization with fixed $\beta$ (in the final upward trend at top-left and centerleft of Fig. 7, in the second downward trend at top-right and center-right of Fig. 7). Therefore, ECO-RP can avoid network congestion in NW1 and NW2 by $\beta$.

From the simulation result, the ECO-RP entity can change link weights without network congestion in NW1 and NW2 when a network operator sets $\alpha$ to 0.03 and $\beta$ to 0.6 or 0.7 .

\section{B. Energy consumption and maximum hop count}

We evaluate ECO-RP from the viewpoint of energy consumption and maximum hop count when we set $\alpha$ to 0.03 and $\beta$ to 0.6 or 0.7 . Figure 8 shows the results for the simulation. Then, we use the following measured values of Cisco GSR 

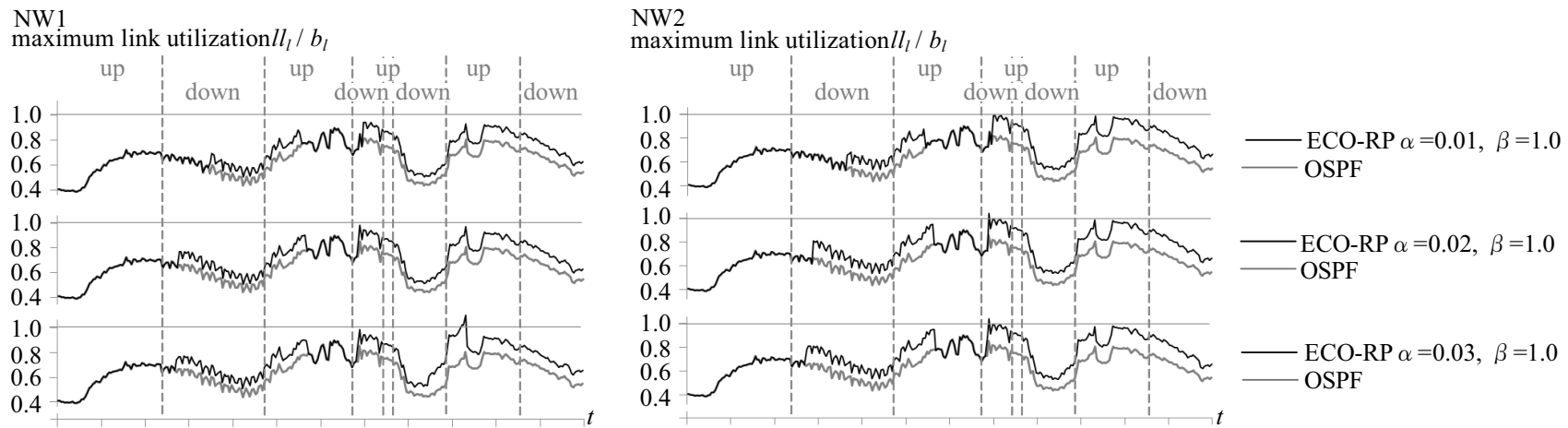

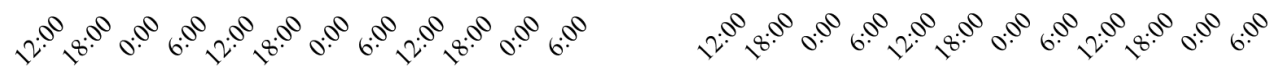

Fig. 6. Maximum link utilization $l l_{l} / b_{l}$ (with variable $\alpha$ and fixed $\beta$ )
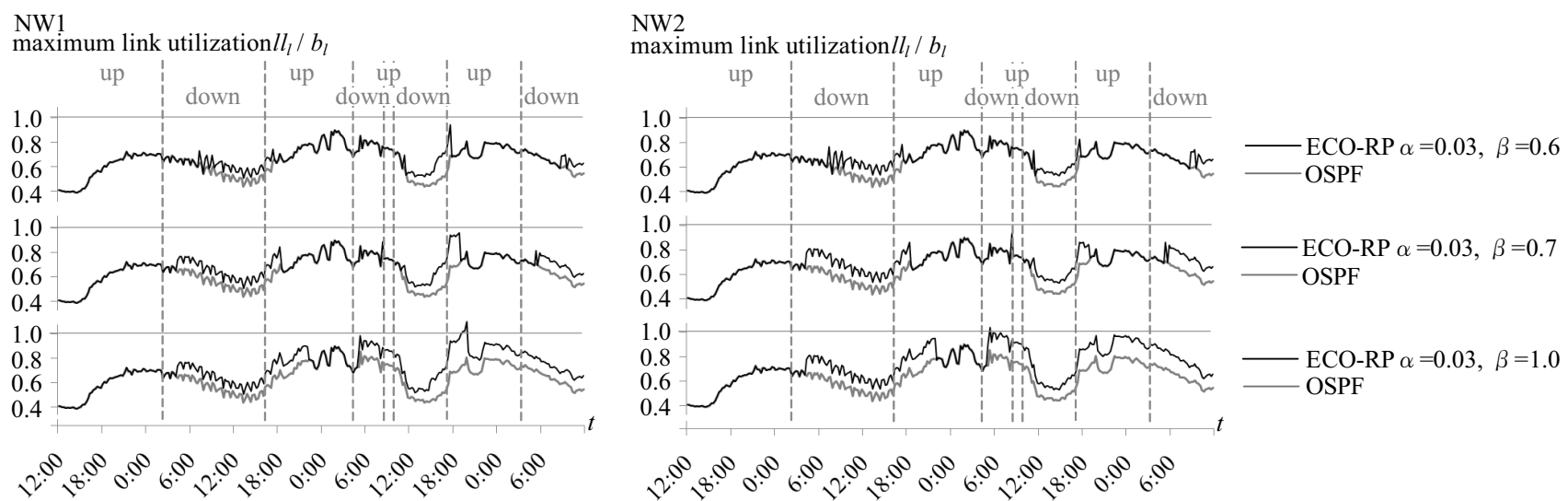

Fig. 7. Maximum link utilization $l l_{l} / b_{l}$ (with fixed $\alpha$ and variable $\beta$ )

12008 for the backbone node and Cisco 7507 for the access node [10] to calculate the $E C$ value:

1) Energy consumption of an active backbone node is 430 watts.

2) Energy consumption of an active access node is 210 watts.

3) Energy consumption of an active link for the backbone node is 70 watts.

4) Energy consumption of an active link for the access node is 25 watts.

We calculate the $E C$ value from the above measured values and the result of Fig. 8:

$E C=$ Num. of active backbone nodes $\times 430$ watts

+ Num. of access nodes $\times 210$ watts

+ Num. of active links of backbone $\times 70$ watts

+ Num. of active links of access $\times 25$ watts

Figure 9 shows the result of $E C$ from the simulation result. The average value of $E C$ over the simulation in
NW1 is 12442.6 watts. On the other hand, if we use the default link weights all the time, which is equivalent to OSPF, the average value of $E C$ is 13270.0 watts. Consequently, ECO-RP can reduce energy consumption by about $6.2 \%(1-12442.6 / 13270.0)$ on average without network congestion if all the nodes are replaced with ECO-RP enabled ones in NW1. Furthermore, the minimum value of $E C$ of the ECO-RP enabled network is 10820.0 watts. Therefore, ECO-RP can reduce energy consumption by about $18.5 \%$ $(1-10820.0 / 13270.0)$ at maximum in NW1. In a similar way, we can see that ECO-RP can reduce energy consumption by about $2.4 \%$ on average and $14 \%$ at maximum without network congestion in NW2. In addition, we can see that there is a trade-off between maximum hop count and the degree to which $E C$ is reduced.

\section{CONCLUSIONS}

This paper presented a new ECO-friendly distributed routing protocol based on OSPF to reduce the energy consumption of routers in ISP networks. In the proposed protocol, the 

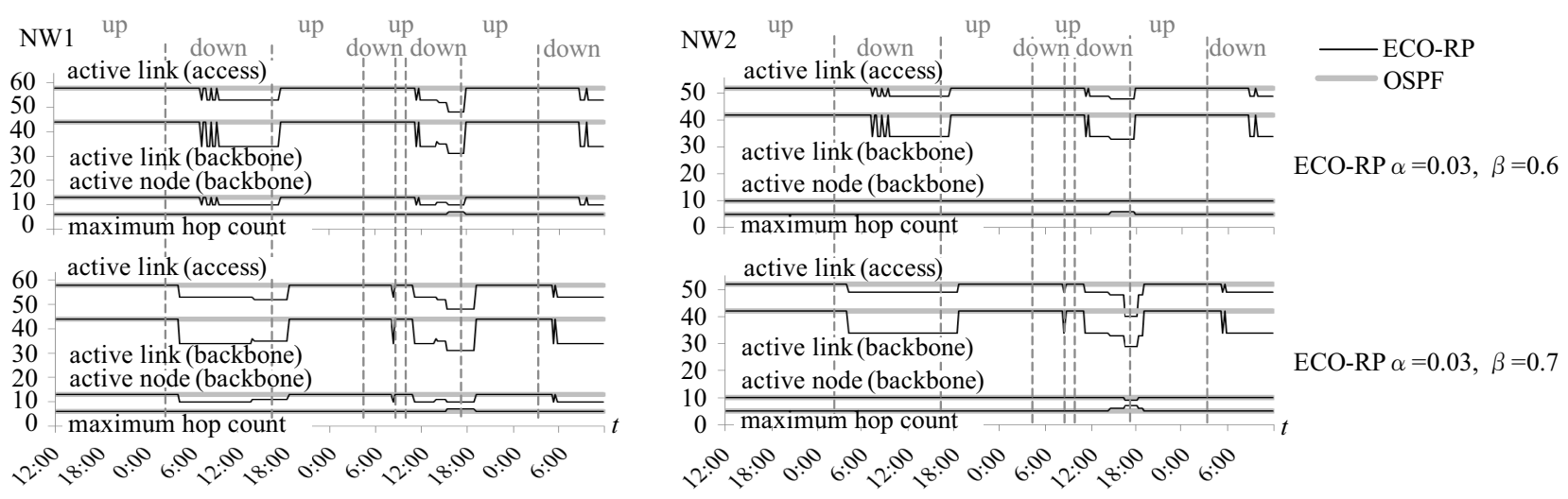

Fig. 8. Number of active nodes, active links, and maximum hop count

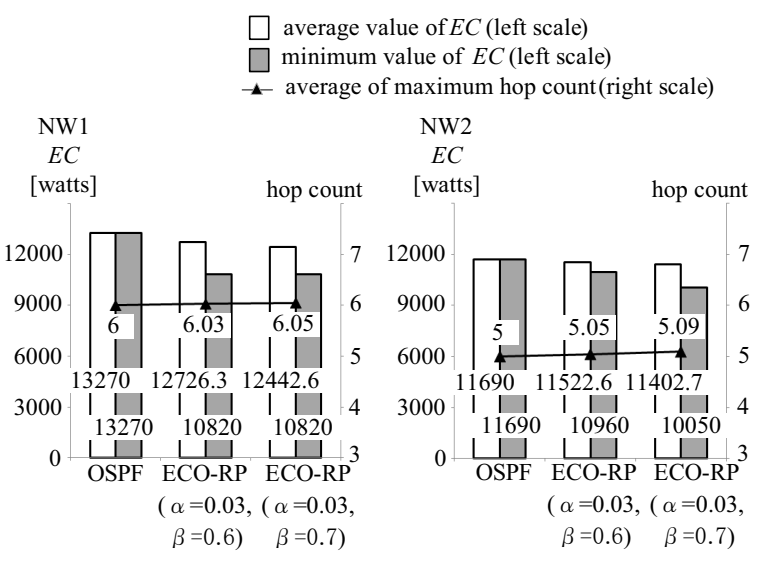

Fig. 9. Energy consumption and hop count

amount of energy consumed by routers is reduced by changing the OSPF link weight in response to the amount of traffic. We showed the details of the proposed protocol. Moreover, to evaluate the performance of the proposed protocol from the viewpoint of energy consumption, we simulated network behavior on two networks generated by a well-known traffic generator. The result showed that the proposed protocol was able to reduce energy consumption by about $18.5 \%$ at maximum without network congestion.

\section{REFERENCES}

[1] A. M. Odlyzko, "Internet traffic growth: Sources and implications, Proceedings SPIE -Optical transmission System and Equipment for WDM Networking II," vol. 5247, pp.1-15, 2003.

[2] M. Gupta and S. Singh, "Greening of the Internet," Proceedings of the ACM SIGCOMM, pp.19-26, 2003.

[3] K. W. Roth, F. Goldstein and J. Kleinman, "Energy Consumption by Office and Telecommunications Equipment in Commercial Building Volume I: Energy Consumption Baseline," National Technical Information Service (NTIS), U.S. Department of Commerce, Springfield, VA22161, NTIS Number: PB2002-101438, 2002.

[4] J. Baliga, K. Hinton and R. S. Tucker, "Energy Consumption of the Internet," COIN-ACOFT, 2007.
[5] J. Baliga, R. Ayre, K. Hinton and R. S. Tucker, "Photonic Switching and the Energy Bottleneck," Photonics in Switching, pp.125-126, 2007.

[6] IEEE 802.3 Energy Efficient Ethernet Study Group. [Online]. Available: http://ieee802.org/3/eee_study/index.html

[7] M. Gupta, S. Grover and S. Shingh, "A Feasibility Study for Power Management in LAN Switches," Proceedings of 12th IEEE International Conference on Network Protocols ICNP 2004, pp.361-371, 2004.

[8] M. Gupta and S. Shingh, "Dynamic Ethernet Link Shutdown for Energy Conservation on Ethernet Links," Proceedings of IEEE International Conference on Communications ICC '07, pp.6156-6161, 2007.

[9] H. Tamura, Y. Yahiro, Y. Fukuda, K. Kawahara and Y. Oie, ”Performance Analysis of Energy Saving Scheme with Extra Active Period for LAN Switches," Proceeding of IEEE Global Telecommunications Conference 2007 GLOBCOM '07, pp.198-203, 2007.

[10] J. Chabarek, J. Sommers, P. Barford, C. Estan, D. Tsiang and S. Wright, "Power Awareness in Network Design and Routing," Proceedings of IEEE the 27th Conference on Computer Communications INFOCOM 2008, pp.1130-1138, 2008.

[11] N. Yamanaka, S. Shimizu and G. Shan, "Energy Efficient Network Design Tool for Green IP/Ethernet Networks," Proceedings of 14th Conference on Optical Network Design and Modeling (ONDM), pp.1-5, 2010.

[12] RFC2328 OSPF Version 2, 1998

[13] D. D. Kouvatsos edt., "Traffic and Performance Engineering for Heterogeneous Networks," River Publishers, ISBN 978-87-92329-16-5, pp.85$107,2009$.

[14] A. Odlyzko, "Data Networks are Lightly Utilized, and will Stay that Way," Review of Network Economics, vol.2, Issue 3, 2003.

[15] A. Nucci, A. Sridharan and N. Taft, "The Problem of Synthetically Generating IP Traffic Matrices: Initial Recommendations," Proceedings of the ACM SIGCOMM, pp.19-32, 2005.

[16] Cisco. OSPF Design Guide. [Online]. Available: http://www.cisco.com/ en/US/tech/tk365/technologies_white_paper09186a00800094e9e.shtml

[17] A. Medina, A. Lakhina, I. Matta and J. Byers, "BRITE: an approach to universal topology generation," Proceedings of Modeling, Analysis and Simulation of Computer and Telecommunication Systems, pp.34-353, 2001

[18] B. Forts and M. Thorup, "Optimizing OSPF/IS-IS weight in a Changing World,'IEEE Jornal on Selected Areas in Communications, vol.20, No.4, pp.756-767, 2002.

[19] S. Uhlig, B. Quoitin, J. Lepropre and S. Balon, "Providing Public Intradomain Traffic Matrices to the Research Community," ACM SIGCOMM, vol. 36, Num. 1, pp.83-86, 2006. 